\title{
Critical Care Research in Elderly Population: An Uncharted Territory
}

\author{
Subhash Todi $^{1}$, Rajat Choudhuri ${ }^{2}$ \\ Indian Journal of Critical Care Medicine (2020): 10.5005/jp-journals-10071-23492
}

In parallel with aging of the general population throughout the world, with the number of individuals aged 80 years or older increasing most rapidly (3.8\% increase/year), more elderly patients are admitted to the intensive care unit (ICU). Definition of "elderly" has been varied in the literature with the three classes that are commonly agreed upon being "young elderly" for patients aged between 65 and 70 or 75 years, "old-old" for patients aged between 70 or 75 and 80 or 85 , and "oldest-old" for patients aged more than 80 or 85 years. Although these terminologies are often used, chronological elderly definition is unreliable because of the interindividual variability in functional status. The difference between "chronological age" and "biological age" has not been very well defined in the literature, but as the latter is not a very robust entry criteria, it is not used for research purposes. Improvements in intensive care in the last few decades have shifted the focus from mortality to quality of life of survivors as a more important outcome measure that is imperative to consider patient-centered outcomes and resource utilization to guide allocation of funds. ${ }^{1,2}$ A recent concept of "Frailty index" (FI) is also gaining popularity among academician. The Fl is a measure of health status of older individuals and serves as a surrogate measure of aging and vulnerability to poor outcomes. It is defined as the proportion of deficits present in an individual out of the total number of agerelated health variables considered. These deficits include diseases, signs, symptoms, laboratory abnormalities, cognitive impairments, and disabilities in activities of daily living. Preclinical frailty before ICU care may be a good marker to identify a population with a high risk of bad outcome after discharge. ${ }^{3} \mathrm{~A}$ broader concept of health-related quality of life ( $\mathrm{HrQOL})$ is mainly based on the World Health Organization's definition of health: "Health is a state of complete physical, mental and social well-being and not merely the absence of disease or infirmity." As a consequence, HrQOL is a subjective concept that depends on individual's perceptions concerning multiple dimensions as physical health (strength, energy), psychological state (good or bad feelings), level of independence (mobility), social relationships (social network and health requirements), life environment (access to care), or personal beliefs (meaning of life). Therefore, its assessment is complex and highly dependent on individual. Various tools such as SF-36 and comprehensive geriatric assessment have been validated to assess quality-of-life issues. The SF-36 questionnaire contains 36 items measuring eight health domains: physical (PF) and social functioning (SF), role limitations due to physical (RP) or emotional problems (RE), mental health (MH), vitality (VT), bodily pain (BP), and general perception of health (GH). Two component scores are physical (PCS) and mental (MCS), are calculated summary scores, where, respectively, the physical domains ( $P F, R P, B P$, and $G H)$ or the mental domains (VT, SF, RE, MH) will account more in the score. A health status index assigns a weight ranging from 0 (in difference
1,2Department of Critical Care, AMRI Hospital, Dhakuria, Kolkata, West Bengal, India

Corresponding Author: Subhash Todi, Department of Critical Care, AMRI Hospital, Dhakuria, Kolkata, West Bengal, India, Phone: +91 33 66260000, e-mail: drsubhashtodi@gmail.com

How to cite this article: Todi S, Choudhuri R. Critical Care Research in Elderly Population: An Uncharted Territory. Indian J Crit Care Med 2020;24(7):500-501.

Source of support: Nil

Conflict of interest: None

between life and death) to 1 (perfect health) corresponding to the overall quality of life.

In this issue of journal, ${ }^{4}$ the authors have studied a cohort of elderly individual aged older than 65 years to assess HrQOL in ICU in this patient population 1 month prior and 3 months post-ICU discharge. Age and level of activity prior to ICU admission were the main determinants of 3-month HrQOL outcome. This observation has a pertinent bearing on clinical decisions of withdrawing and withholding life support in the elderly individuals. The immediate acuity of the disease is sometimes the main determinant of making such decision with the notion that the quality of life will be much worse in these individuals even if they come out of the acute crisis. This and other studies have consistently shown that preadmission quality of life is one of the major determinants of post-ICU HrQOL and should be factored in while taking end-of-life decisions in these individuals. ${ }^{5,6}$ Thus, despite high chronological age, if $\mathrm{HrQOL}$ was satisfactory preadmission, continuing ICU care may not be inappropriate and "futility" criteria as far as HrQOL is concerned may not be applicable to these patients and will be comparable to their younger counterpart. The "psychological" and "social" aspects of the HrQOL will be variable depending on the geographical location, social structure, and cultural influences and cannot be extrapolated to our settings. In developing countries, due to lack of comprehensive post-ICU physical rehabilitation facilities, the physical domain of HrQOL may not improve so much, whereas a large social support may maintain psychological aspects of HrQOL. ${ }^{7-9}$

Further research needs to be done to answer the question as to what is the optimum time period post-ICU discharge to ascertain HrQOL. This can only be ascertained by following longitudinally a cohort of such patients to follow their natural course of recovery and the time to reach a plateau level. Very long follow-up will not be a realistic proposition in the elderly population. Furthermore, a detailed evaluation of different aspects of HrQOL also needs to be determined, as the physical and psychological qualities may be discordant and societal, and other factors determining the impact on individual HrQOL will be important in order for 
appropriate intervention policy to make an impact on the outcome. The trajectories of improvement or decline in $\mathrm{HrQOL}$ should also be studied by predictive analysis. A distinction should also be made between "young elderly" and the "oldest-old," with the former having a better prognosis compared to their younger counterparts. Paradoxically, it has also been shown that some elderly patients even perceive a better quality of life post-discharge, conceivably due to more attention to their nutrition and physical rehabilitation which was neglected preadmission. Limitation of a qualitative research with survey questionnaire answered by a caregiver or next of kin may not be very accurate. The emotional dimension seems to be assessed less accurately by relatives than the physical one. In summary, HrQOL of life post-ICU discharge as a parameter of decision-making in the ICU for elderly population should take into account their preadmission functionality. Preadmission HrQOL may be a surrogate marker of "Biological" age of the elderly as opposed their "chronological" age and should be given due importance.

\section{References}

1. Conti M, Merlani P, Ricou B. Prognosis and quality of life of elderly patients after intensive care. Swiss Med Wkly 2012;142:w13671. DOI: 10.4414/smw.2012.13671.

2. Gurjar M, Azim A. Quality of life after intensive care unit discharge in a tertiary care hospital in India: cost effectiveness analysis. Indian J of Crit Care Med 2019;23(3):122-126. DOI: 10.5005/ jp-journals-10071-23133.

3. Flaatten $\mathrm{H}$, De LangeDW, Morandi A, Andersen FH, Artigas A, Bertolini G, et al. The impact of frailty on ICU and 30-day mortality and the level of care in very elderly patients ( $\geq 80$ years). Intensive Care Med 2017;43(12):1820-1828. DOI: 10.1007/s00134-017-4940-8.

4. Zineb Z, Abidi K, Dendane T, Kettani MNZ, Iraqi A, Belayachi J, et al. Health-related quality of life evaluated by MOS SF-36 in the elderly patients 1 month before ICU admission and 3 months after ICU discharge. Indian J Crit Care Med 2020;24(7):531-538.

5. Atramont A, Lindecker-Cournil V, Rudant J, Tajahmady A, DrewniakN, Fouard $A$, et al. Association of age with short-term and long-term mortality among patients discharged from intensive care units in France. JAMA Network Open 2019;2(5):e193215. DOI: 10.1001/ jamanetworkopen.2019.3215.

6. Sacanella E, Pérez-Castejón JM, Nicolás JM, Masanés F, Navarro $\mathrm{M}$, Castro $\mathrm{P}$, et al. Functional status and quality of life 12 months after discharge from a medical ICU in healthy elderly patients: a prospective observational study. Crit Care 2011;15(2):R105. DOI: 10.1186/cc10121.

7. Lee SH, Kim JY, Kim TH, Ju SM, Yoo JW, Lee SJ, et al. Retrospective analysis of long-term survivalin very elderly (age $\geq 80$ ) critically III patients of a medical intensive care unit at a tertiary care hospital in Korea. Tuberc Respir Dis 2020;83(3):242-247. DOI: 10.4046/ trd.2019.0032.

8. Andersen $\mathrm{FH}$, Flaatten $\mathrm{H}$, Klepstad $\mathrm{P}$, Romild U, Kvåle R. Long-term survival and quality of life after intensive care for patients 80 years of age or older. Ann Intensive Care 2015;5(1):53. DOI: 10.1186/s13613015-0053-0.

9. Guidet B, Leblanc G, Simon T, Woimant M, Quenot JP, Ganansia O, et al. Effect of systematic intensive care unit triage on long-term mortality among critically III elderly patients in France: a randomized clinical trial. JAMA 2017;318(15):1450-1459. DOI: 10.1001/jama.2017. 13889. 